\title{
Surgical Strategies for Spondylodiscitis due to Lumbar Disc Surgery
}

\author{
Ahmet KUCUK ${ }^{1}$, Mustafa KARADEMIR ${ }^{2}$, Abdulfettah TUMTURK ${ }^{1}$, Halil ULUTABANCA ${ }^{1}$, Baris Derya ERCAL ${ }^{3}$, \\ Serkan SENOL ${ }^{4}$, Ahmet MENKU ${ }^{1}$
}

${ }^{1}$ Erciyes University, School of Medicine, Department of Neurosurgery, Kayseri, Turkey

${ }^{2}$ Afyon State Hospital, Neurosurgery Clinic, Afyon, Turkey

${ }^{3}$ Erciyes University, School of Medicine, Department of Microbiology, Kayseri, Turkey

${ }^{4}$ Erciyes University, School of Medicine, Department of Radiology, Kayseri, Turkey

\section{ABSTRACT}

AIM: Despite different surgical treatment protocols at different centers for spondylodiscitis due to lumbar surgery, there is no consensus on its surgical indications. In this study, we aimed to clarify the steps to be followed in the management and treatment of postoperative spondylodiscitis.

MATERIAL and METHODS: The data of 20 cases with postoperative spondylodiscitis were evaluated. C-reactive protein (CRP) was used for diagnosis and follow-up. According to culture results of the infected material obtained from the operated cases, appropriate antibiotic treatment was initiated. In non-operated cases, parenteral empirical antibiotic treatment was implemented. Surgical treatment was planned for cases with clinical and radiological instability, abscess on imaging and those who were nonrespondent to empirical antibiotic treatment. For the cases that clinically recovered and had normal CRP levels, oral antibiotic treatment was continued after parenteral antibiotic treatment.

RESULTS: Of the cases; 13 were male (65\%) and 7 were femals (35\%). The mean age was 56.3 years (32-74). The most prevalent complaints in referral were waist and leg pain. Except one, all cases had increased CRP levels. All patients had spondylodiscitis on magnetic resonance imaging. Seven had radiological and clinical instability and 3 had epidural abscess. The most commonly growing microorganism in culture was Staphylococcus aureus. Surgical treatment was applied to seven cases and medical treatment to 13 cases.

CONCLUSION: In cases with waist pain in the postoperative period, the first potential diagnosis to be considered is spondylodiscitis. Surgical treatment should be implemented for cases resistant to empirical antibiotic treatment, with abscess on imaging, or with lumbar instability.

KEYWORDS: Spondylodiscitis, Spinal surgery, Postoperative period

\section{INTRODUCTION}

$\mathrm{T}$ The complication rates of spinal surgery have increased in recent years together with the increase in spinal surgery (15). The number of postoperative spondylodiscitis cases has also increased due to the increase both in surgery for the aged population and in the number of immunosuppressed cases $(4,6)$.
Whereas advanced age, immunosuppression, spinal trauma, chronic renal failure and diabetes mellitus are the individual risk factors that cannot be changed in infection pathogenesis, obesity, smoking, invasive catheters and prolonged hospital stay are among the factors that can be changed. Exposure of the surgical area to the radiation, malignancy and chemotherapy also increase the postoperative infection risk (9). 
In the pathogenesis, the hematogenous spread of microorganisms out of the genitourinary or gastrointestinal system as well as the microorganisms of the skin flora due to inadequate asepsis are blamed (1).

End plate injury, the cauterization of many small vessels, the presence of hematoma in the surgical area and necrotic areas due to surgery create an ideal environment for the bacteria to settle down and proliferate (10).

Postoperative spondylodiscitis is an infectious disease that can only be treated with difficulty. A multidisciplinary approach including appropriate microbiological and radiological diagnosis, proper antibiotic treatment and, if needed, surgical intervention is required. In the majority of cases, surgery is needed to confirm diagnosis, to limit the infection, and to wash the surgical area (2).

Although the diagnosis of postoperative spondylodiscitis can be made clearly via microbiological and radiological methods, its management and treatment are unclear and controversial. Some authors recommend that most cases should be surgically treated $(10,14)$, and others believe that this is not necessary unless conservative treatment fails or an abscess or epidural mass is present $(7,17)$.

In the current study, we aimed to clarify the steps to be followed in the appropriate diagnosis and effective treatment of postoperative spondylodiscitis, which give rise to excessive cost due to the antibiotic treatment, affect the quality of life of the cases negatively, and cause a loss in the workforce.

\section{- MATERIAL and METHODS}

We evaluated 20 cases with postoperative spondylodiscitis among the 1829 cases that underwent lumbar microdiscectomy between 2008 and 2013 by the same surgeon at Erciyes University, Faculty of Medicine, Department of Neurosurgery. The primary surgical diseases of the patients, the time when the complaints began, the complaints when referred to the hospital and examination findings, C-reactive protein (CRP) levels, radiological findings, hospitalization, the antibiotic treatment and duration, and the conditions under which they underwent the second surgery were examined in detail.

CRP levels were measured at the time of referral and weekly afterward. The levels were evaluated by a specialist on infectious diseases and empiric antibiotic treatment was initiated for the cases that would not undergo surgical treatment.

Dynamic (hyperflexion and hyperextension) radiographies were performed to determine whether spinal instability was present or not, and magnetic resonance imaging (MRI) was performed to find out whether spondylodiscitis and spinal epidural abscess were present or not. In cases with spinal instability, computerized tomography (CT) was used before the second surgery to better evaluate the bone structure and end plate injury.

In cases without instability and epidural abscess, empiric antibiotic treatment with Ciprofloxacin 2x200 mg intravenous (iv) + Teicoplanin $2 \times 400 \mathrm{mg}$ iv ( $1 \times 400 \mathrm{mg}$ after the $3^{\text {rd }}$ day) for 3-6 weeks or Imipenem $4 \times 500 \mathrm{mg}$ iv + Teicoplanin $2 \times 400 \mathrm{mg}$ iv (1x400 mg after the $3^{\text {rd }}$ day) for 3- 6 weeks were initiated by a specialist on infectious diseases. The response of the cases to antibiotic therapy was evaluated by weekly CRP controls in the first month. Antibiotic therapy was changed when there was no decrease in CRP levels. Oral antibiotic treatment was started when CRP levels decreased to $33 \%$ at the end of 3-6 weeks of antibiotic treatment and/or there was no hyperintensity on MRI.

Cases with instability or epidural abscess underwent surgical treatment. The cases with instability were stabilized by transpedicular instrumentation. In the cases with epidural abscess, the abscess was drained. In the cases that needed closed irrigation drainage, $1000 \mathrm{ml} \mathrm{NaCl} 0.9 \%$ including 500 $\mathrm{mg}$ rifampicin/24 $\mathrm{h}$ was administered for 3 days. The gram staining and culture results of the leakage material in the surgical area or of the infected material taken at surgery were evaluated by a microbiologist and a specialist on infectious diseases. The cases undergoing surgical treatment were administered empiric antibiotic treatment until the appropriate antibiotic regime was determined according to the culture results and antibiogram after the surgery.

\section{RESULTS}

There were 11 (55\%) males and 9 (45\%) females. The mean age was 56.3 years (32-74). All the cases had lumbar disc hernia. The mean duration for the complaints to begin after surgery was 54.9 days (4-175).

\section{Clinical Results}

Nine of the cases had back pain complaints, extending to paravertebral area, increasing with movement and decreasing with rest. The pain extended to the paravertebral site. Four of the cases had unilateral leg pain and two had bilateral leg pain accompanied by back pain. One case had leg pain only and one case had hip pain only. The pain generally did not respond to non-steroidal anti-inflammatory drugs and had an increasing character. In two of the cases with pain complaint, there was a leakage from the wound site. One case that did not have pain was admitted to our clinic with leakage from the surgical area. There was no motor deficiency on examination. In three of the cases, there was a sensory deficiency of various degrees.

\section{Laboratory Results}

Routine whole blood count and biochemical tests, and CRP measurements were requested and CRP levels increased in nearly all the cases. CRP was within normal limits in only one case. At the end of the six month-follow-up, there were two cases with decreasing CRP levels but they did not return to normal. In the follow-up of these cases, the CRP level turned out to be normal at the first year of treatment.

\section{Radiological Results}

Dynamic radiographs and lumbar MRI were used in all cases. Cases with lumbar instability and those who would undergo 
surgery for stabilization underwent lumbar CT and sagittal reconstruction. All the cases had spondylodiscitis. Of the seven cases undergoing surgery, six had lumbar instability and end plate irregularity, and three had epidural abscess. Spondylodiscitis was detected in L4-5 in 13 of the cases (65\%), in L5-S1 in four of the cases (20\%), in L3-4 in two of the cases (10\%), and in L2-3 in one of the cases (5\%).

\section{Microbiological Results}

Microbiological samples were taken from the surgical area in seven cases during the second surgery and from the leakage on the surgical site in three cases. The most prevalent microorganisms according to the culture results were Staphylococcus aureus in three cases (42\%). Escherichia coli in one (14.5\%) case, Staphylococcus epidermidis in one (14.5\%) case, Streptococcus viridans in one (14.5\%) case and A. baumanii in one (14.5\%) case.

\section{DISCUSSION}

Postoperative spondylodiscitis, first described by Turnbull in $1953(20)$, is a rare $(8,9,12)$ but serious and increasing complication of spinal surgery (17). The prevalence in the literature $(1,5,11,18)$ is between $0.2 \%$ and $4.0 \%$. In the last five years, our surgical team has operated on a thoracic and/or lumbar site in 1829 cases and postoperative spondylodiscitis developed in 20 of these cases (1.09\%).

Direct inoculation of the infective agent during surgery (9) and the access to the genitourinary or gastro-intestinal systems through the hematogenous route are blamed in the pathogenesis of postoperative spondylodiscitis (1).

Recurrent back and leg pains in the postoperative period may be the first signs of postoperative spondylodiscitis. This pain often increases with standing, physical effort, and walking; and decreases with rest and lying down. Back or leg pain was the first symptom in our cases. These cases should be followedup more frequently, and the localization, intensity and change of the pain should be assessed. Besides, the presence of a neurological deficiency should be evaluated. General status, posture, walking and fever should also be evaluated. The operation site should be checked. Erythema, leakage, a mass, and opening of the wound should be considered as signs of an infection. If an orthosis or corset has been advised, its appropriateness should be evaluated (2).

The aim of radiological evaluation in cases with back and leg pain in the postoperative period is to evaluate the sufficiency of surgery, instability, the presence of discitis and to what extent it affected the spine, and to evaluate the presence of an epidural abscess. Coronal and sagittal plan alignment, intervertebral vertebral height, end-plate erosions and the physiologic and pathologic inclinations are evaluated By lumbar radiographies. Dynamic radiographies aid to determine the presence of instability. In seven of our cases, we also detected instability with imaging techniques.

$\mathrm{CT}$ is very beneficial in evaluating the bone structures. Bone destruction, vertebral body deflation, end-plate irregularities and liquid collection may be seen in spondylodiscitis. An epidural or paravertebral abscess is seen as peripheral contrast-enhanced and smooth-bordered lesions (2).

MRI is the most trustworthy and specific imaging technique for spondylodiscitis (13). The complaints, examination and laboratory results of the cases may suggest spondylodiscitis, but the main diagnostic technique is MRI. Hypointensity on T1W image and hyperintensity on T2W image in the intervertebral space are seen on MRI. Following gadolinium injection, contrast enhancement in the intervertebral space or peripheral contrast enhancement and a clear bordered abscess focus may be seen (18). There is sclerosis and destruction in the end-plate, a decrease in the intervertebral space, and edematous signal changes in the vertebral bodies neighboring the disc (10). MRI was planned for all of our cases, and there was an epidural abscesses in three of them, of various sizes.

Although the optimal method in the diagnosis of spondylodiscitis is the combination of radiological imaging techniques, positron-emission tomography (PET) and PET-CT use in addition to the conventional single photon emission computerized tomography used for the diagnosis of skeletal-muscle system infections has increased in recent years (19).

CRP is a protein that increases with an acute or chronic inflammatory response. It is generally used for diagnostic purposes for infections $(16,21)$. All our cases except one had increased CRP levels. CRP is used not only for diagnostic purposes, but to monitor infections as well. CRP levels generally decrease with the use of antibiotics. However, CRP levels decreased nearly one year after antibiotic therapy in two of our cases. In Bavinzski's study including 17 cases, CRP levels were high in one case despite the use of antibiotics (3). In the rest of our cases, CRP levels decreased to normal with surgical and/or medical treatment.

In order to decide on surgical treatment and the kind of surgical treatment in spondylodiscitis, the evaluation of clinical, laboratory and radiological findings should be performed together.

Surgical treatment is required for cases with instabilities in dynamic radiographies and the physical examination, with high levels of CRP despite antibiotic therapy, and with an abscess appearance on CT or MRI.

The purpose of surgical treatment is to drain an abscess if it exists, to treat instability, debride necrotic tissues, and to place an irrigation system into surgical area (14). In our six cases with instability, stabilization was ensured with transpedicular screws. In three of these cases, there was also an epidural abscess. In three cases without instability, there was an epidural abscess. These abscesses were drained during surgery.

What is more important than antibiotic therapy and surgical treatment in the precise and prompt diagnosis of spondylodiscitis is not to cause spondylodiscitis. This may be achieved by taking appropriate precautions before, during and after the operation. In the preoperative period, one must ensure that the patient takes a shower the day before the operation, and 
the number of visitors should be minimized and if possible no visitors should be allowed. The case should be shaved on the operation table before the operation without impairing the integrity of the skin. It is generally accepted to apply prophylactic antibiotics thirty minutes before the operation. Immediately before the operation, the surgical site should be washed with solutions including antibiotics for 3-5 minutes to remove the skin flora. After surgical site isolation, the use of sterile drapes will prevent the skin staying exposed during the operation. A small surgical opening, minimal invasive approaches, and releasing the distractor in prolonged operations and additional antibiotic use are intraoperative precautions. In the postoperative period, the sutures are recommended to be taken out on the $7^{\text {th }}$ day and the patient is advised to take a shower afterwards.

\section{CONCLUSION}

Spondylodiscitis due to disc surgery is a rare but serious complication. In cases with recurrent back and leg pains in the postoperative period, spondylodiscitis should be suspected of and CRP, MRI and culture should be requested. In the majority of cases with postoperative spondylodiscitis, intravenous antibiotic use and immobilization will be sufficient, while surgical treatment is required in the minority of nonrespondent cases with instability.

\section{- REFERENCES}

1. Adam D, Pevzner E, Gepstein R: Comparison of percutaneous nucleoplasty and open discectomy in patients with lumbar disc protrusions. Chirurgia (Bucur) 108: 94-98, 2013

2. Barrey C, Launay O, Freitas E, Michel F, Laurent F, Chidiac C, Perrin G, Ferry T: The follow-up of patients with postoperative infection of the spine. Eur J Orthop Surg Traumatol 23 Suppl 1: 29-34, 2013

3. Bavinzski G, Schoeggl A, Trattnig S, Standhardt H, Dietrich W, Reddy M, Al-Schameri R, Horaczek A: Microsurgical management of postoperative disc space infection. Neurosurg Rev 26: 102107, 2003

4. Bible JE, Biswas D, Devin CJ: Postoperative infections of the spine. Am J Orthop (Belle Mead NJ) 40: 264-271, 2011

5. Boden SD, Davis DO, Dina TS, Sunner JL, Wiesel SW: Postoperative diskitis: Distinguishing early MR imaging findings from normal postoperative disk space changes. Radiology 184 765-771, 1992

6. Chaichana KL, Bydon M, Santiago-Dieppa DR, Hwang L, Mc Loughlin G, Sciubba DM, Wolinsky JP, Bydon A, Gokaslan ZL, Witham T: Risk of infection following posterior instrumented lumbar fusion for degenerative spine disease in 817 consecutive cases. J Neurosurg Spine 20: 45-52, 2014
7. D'Agostino C, Scorzolini L, Massetti AP, Carnevalini M, d'Ettorre G, Venditti M, Vullo V, Orsi GB: A seven-year prospective study on spondylodiscitis: Epidemiological and microbiological features. Infection 38: 102-107, 2010

8. Demiraran Y, Dosoglu M, Yavuz C, Akyuz F: Spondilodiscitis and lumbar epidural abscess occurring after orthopedic epidural anesthesia: A Case report. Turk Neurosurg 16: 208-211, 2006

9. Gerometta A, Bittan F, Olaverri JCR: Postoperative spondilodiscitis. International Orthopaedics (SICOT) 36: 433-438, 2012

10. Hamdan TA: Postoperative disc space infection after discectomy: A report on thirty-five patients. International Orthopaedics (SICOT) 36: 445-450, 2012

11. Jimenez-Mejias ME, de Dios CJ, Sanchez-Lora FJ, PalominoNicas J, Reguera JM, Garcia de la Heras J, Garcia-Ordonez MA, Pachon J: Postoperative spondylodiscitis: Etiology, clinical findings, prognosis, and comparison with nonoperative pyogenic spondylodiscitis. Clin Infect Dis 29: 339-345, 1999

12. Karagoz Guzey F, Emel E, Baş NS, Alatas I, Sel B: Lumbar spondylodiscitis caused by Morganella morganii. Turk Neurosurg 14: 57-62, 2004

13. Khan IA, Vaccaro AR, Zlotolow DA: Management of vertebral discitis and osteomyelitis. Orthopedics 22: 758-765, 1999

14. Nasto LA, Colangelo D, Rossi B, Fantoni M, Pola E: Post-operative spondylodiscitis. Eur Rev Med Pharmacol Sci 16 Suppl 2: 50-57, 2012

15. Nunez-Pereira S, Rodriguez-Pardo D, Pellise F, Pigrau C, Bago J, Villanueva C, Caceres E: Postoperative urinary tract infection and surgical site infection in instrumented spinal surgery: Is there a link? Clin Microbiol Infect 20:768-773, 2014

16. Osmon DR, Berbari EF, Berendt AR, Lew D, Zimmerli W, Steckelberg JM, Rao N, Hanssen A, Wilson WR: Diagnosis and management of prosthetic joint infection: Clinical practice guidelines by the infectious diseases society of America. Clin Infect Dis 56: 1-10, 2013

17. Rawlings CE, Wilkins RH, Gallis HA, Goldner JL, Francis R: Postoperative intervertebral disc space infection. Neurosurgery 13: 371-376, 1983

18. Silber JS, Anderson DG, Vaccaro AR, Anderson PA, McCormick $P$ : Management of postprocedural discitis. Spine J 2:279-287, 2002

19. Steven P, Steve R, Cho Y: The role of PET in the evaluation of musculoskeletal infections. Semin Musculoskelet Radiol 18:166174,2014

20. Turnbull F: Postoperative inflammatory disease of lumbar discs. J Neurosurg 10:469-473, 1953

21. Zimmerli W: Clinical practice. Vertebral osteomyelitis. N Engl J Med 362:1022-1029, 2010 